\title{
Refractory tenosynovitis with 'rice bodies' in the hand due to Mycobacterium intracellulare
}

\author{
Ho Namkoong ${ }^{1} \cdot$ Keizo Fukumoto $^{2} \cdot$ Igen Hongo $^{3} \cdot$ Naoki Hasegawa $^{4} \oplus$
}

Received: 27 July 2015 / Accepted: 15 September 2015 / Published online: 26 September 2015

(c) The Author(s) 2015. This article is published with open access at Springerlink.com

Fig. 1 a Swelling and tenderness of the left thumb and thenar before the first operation (red arrow). b 'Rice bodies' were observed during the first open drainage performed for treating tenosynovitis. c ${ }^{18} \mathrm{~F}$-fluorodeoxyglucose positron emission tomography/computed tomography showed intense uptake around the metacarpophalangeal joint, before the third drainage. $\mathbf{d}$ Intraoperative findings at the third drainage showed synovial thickening and synovial fluid retention. By this time, the 'rice bodies' had resolved (red arrow)
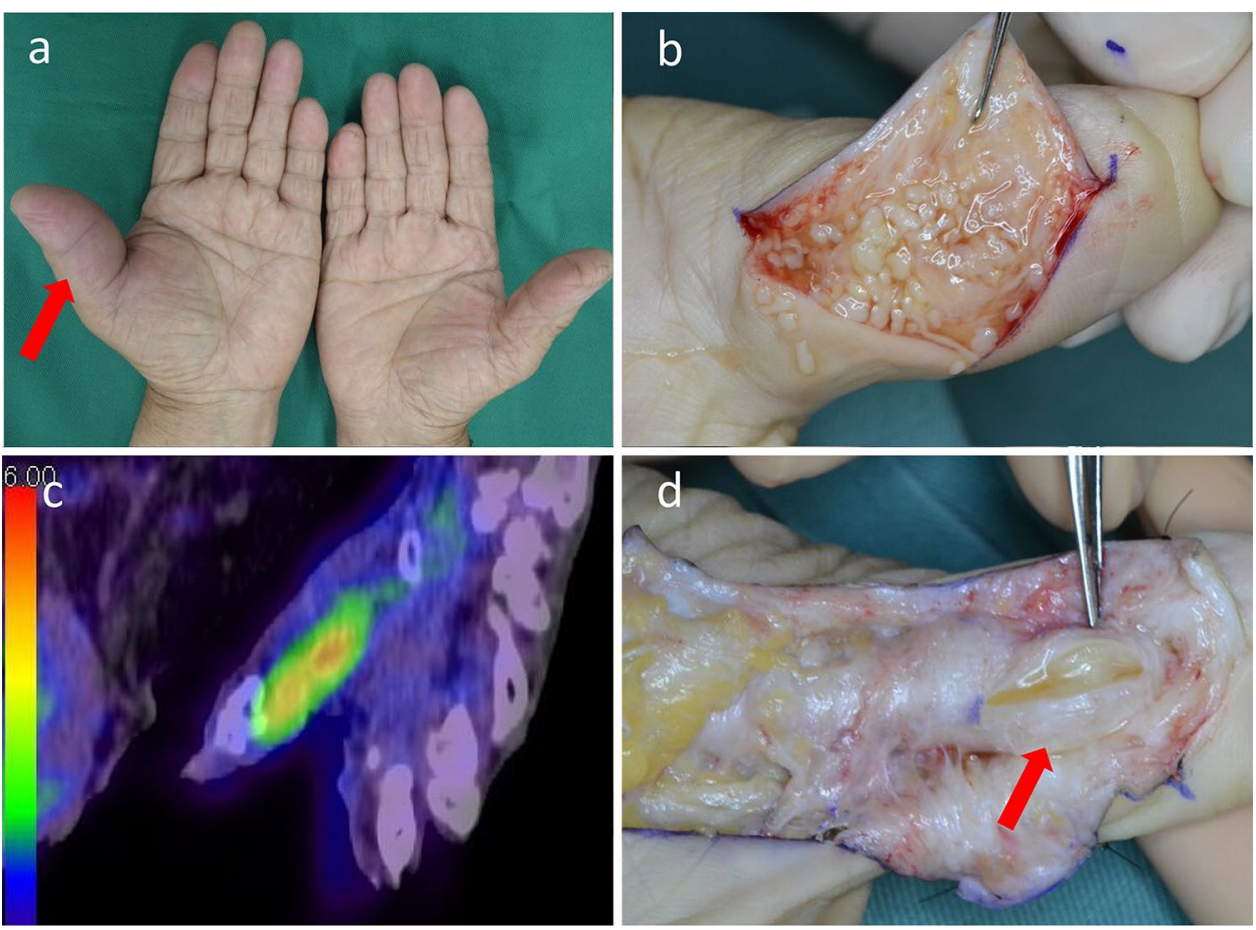

Naoki Hasegawa

n-hasegawa@z8.keio.jp

1 Division of Pulmonary Medicine, Department of Medicine, Keio University School of Medicine, Shinjuku, Tokyo, Japan

2 Saitama Hand Surgery Institute of Saitama Seikeikai Hospital, Higashimatsuyama, Saitama, Japan

3 Division of Infectious Diseases, Musashino Red Cross Hospital, Musashino, Tokyo, Japan

4 Center for Infectious Disease and Infection Control, Keio University School of Medicine, Shinjuku, Tokyo 160-8582, Japan

\section{Legend}

A 76-year-old man presented with a 2-month history of swelling and tenderness of the left thumb and thenar. The patient had type 2 diabetes mellitus. He had been bitten by a dog on the left hand 1 year previously, and the wound had healed without treatment. He was initially diagnosed with non-infectious tenosynovitis and received steroid injections repeatedly (Fig. 1a). Thereafter, open diagnostic-drainage was performed, and the presence of 'rice bodies' was visually noted in the hand (Fig. 1b). 
Based on the pathological finding of granuloma and positive specimen culture for Mycobacterium intracellulare, he was diagnosed with tenosynovitis due to Mycobacterium avium complex (MAC). While his symptoms initially improved by isoniazid, rifampicin, and ethambutol, the redness and tenderness around the left wrist gradually worsened at 6 months after the first operation. Then therapeutic-drainage was performed again, and the regimen was changed to clarithromycin, rifampicin, ethambutol, and sitafloxacin after the introduction to our department. After 1 year, however, a nodule developed around the metacarpophalangeal joint, associated with an intense uptake on ${ }^{18} \mathrm{~F}$-fluorodeoxyglucose positron emission tomography/computed tomography (Fig. 1c), implying the residual inflammation. Therapeutic-drainage was performed again (Fig. 1d), and he is now in remission under antimicrobial chemotherapy.

MAC tenosynovitis is a refractory infectious disease, reported more commonly in Asians [1-3]. Most patients have an injury history and often require multiple operations as in this case [1-4]. The presence of 'rice bodies' is a characteristic intraoperative finding as well as tuberculosis $[3,5]$. Although the appropriate duration of chemotherapy is unclear, past studies recommended a 1-2-year treatment period [4]. When seeing cases present with refractory tenosynovitis, MAC tenosynovitis should be considered in the differential diagnosis.

\section{Compliance with ethical standards}

\section{Conflict of interest None.}

Open Access This article is distributed under the terms of the Creative Commons Attribution 4.0 International License (http://creativecommons.org/licenses/by/4.0/), which permits unrestricted use, distribution, and reproduction in any medium, provided you give appropriate credit to the original author(s) and the source, provide a link to the Creative Commons license, and indicate if changes were made.

\section{References}

1. Choi JJ, Ban WH, Jung YH, Bae MN, Baek IW, Kim KJ, Cho CS. Mycobacterial tenosynovitis of the hand in a patient with systemic lupus erythematosus. Int J Rheum Dis. 2013;16:364-6.

2. Yoon HJ, Kwon JW, Yoon YC, Choi SH. Nontuberculous mycobacterial tenosynovitis in the hand: two case reports with the MR imaging findings. Korean J Radiol. 2011;12:745-9.

3. Chan HT, Tseng CC, Chen PY, Chao CM, Lai CC. Rice body-Mycobacterium intracellulare tenosynovitis. QJM. 2014;107:395.

4. Hellinger WC, Smilack JD, Greider JL Jr, Alvarez S, Trigg SD, Brewer NS, Edson RS. Localized soft-tissue infections with Mycobacterium avium/Mycobacterium intracellulare complex in immunocompetent patients: granulomatous tenosynovitis of the hand or wrist. Clin Infect Dis. 1995;21:65-9.

5. Weber E, Gagneux-Brunon A, Jacomo V, Rousselon T, Lucht F, Botelho-Nevers E. Tenosynovitis: a rare presentation of tuberculosis better known by hand surgeons than infectious diseases specialists. Infection. 2015;43:261-6. 